\title{
A Study of Cyber video Technology Application Based on Cloud Computing Platform
}

\author{
ZHOU Yanfang ${ }^{1, a}$ \\ ${ }^{1}$ Northern Beijing Vocational Education Institute, Beijing 101400, China \\ a nmzhouyanfang@163.com
}

Keywords: Clouding computing; Cyber video service; Cloud storage; Cloud architecture

\begin{abstract}
With the fast development of the computer technology and social developing needs, the network has experienced quite a lot of changes and the usage of it has also entered into more areas. As one mature technical service which is widely accepted by the public, cyber video has the inevitability and feasibility to associate with cloud computing technology. This article analyzes the features of cloud cyber video platform and its technical needs. It proposes an infrastructure that explained how to use cloud computing technology to build a service platform which is flexible, reliable, energy conservation and security.
\end{abstract}

\section{Introduction}

At present, cloud computing has stepped into a new developing state, that is to say cloud computing is a new revolution in information era. Many experts think that cloud computing will change the technical basis, even to affect the whole industrial pattern. How to use the theory and technology which is related to cloud computing; How to exert more core competencies in the situation of triple play, It's not only an rare opportunity but also a tough challenge for those who work on radio and TV.

The triple play is not only an inevitable trend of Internet media, but the future of China radio and TV. [1]To vigorously develop a new program form which turns toward multiple platforms, multiple media and multiple terminals is a real issue lying before broadcasting, TV station and new media unit. And it can be transformed by content resource management system's cloud computing.

\section{From “cloud computing” to "cloud video"}

With the rapid development of the media industry, all kings of new media technology came out one after the other. The traditional business needs to have more chances to realize in the way of using normalization and networking.

\section{The Concept of Cloud Computing}

Clouding Computing is an emerging pattern that service use the way of demanding delivery. [2]This makes a great deal of computing, storage, networking and others become resource pooling. And this lets every kind of application systems have access to various resources and services according to the demand.

(1) Clouding Computing is the product of integration of a traditional computing technology and network technology. The essence of Clouding Computing is the extension of host-client mode.

(2) Cloud Computing developed on the basis of parallel computing distributed computing and network computing. [3]It is the result of fusion and evolution of those concepts, including virtualization, utility computing, making infrastructure as service, making platform as service and making software as service. And it provides the required of computing power, storage space, software function, information service and etc.

Generally speaking, Cloud Computing is a kind of service delivery and usage patterns which was easy to expand, cheap and has the advantages of large scale and virtualization.

The Main Features of Cloud Computing

(1)Cloud Computing absorbed the advantages of the previous generation of computer models, but there are still difference between them, even bring the previous computer models in it self's 
framework. In essence, Cloud Computing can make users get storage, computing, database and other services by remote connection.

(2)Cloud Computing has the advantages of getting what you actually need, resource optimization, scalability. Exactly it can help Radio and TV establishes information system networking of low cost and management optimization for a short while. Specifically, Cloud Computing can help Radio and TV to achieve more abundant media resources, to serve in better performance, to manage more precise, to provide users with a better experience.

(3)Cloud Computing technology is the use of virtualization technology. This makes full use of idle resources, provide service instance with independent and secure runtime environment. [4]Through virtualization technology, Cloud Computing technology distributes various types of virtual resources according to need so that it can satisfy the dynamic virtual resources.

(4)The main feature od Cloud Computing is that the system has large data sets. Based on the data sets, it can provide users with service.

\section{The Application Prospect of “Cloud Computing” in Radio and Television Industry}

Currently, the Cloud Computing technology and the service on the basis of Cloud Computing have become the research priorities of television industry. Cloud Computing is regarded as a revolution in the technology industry. It will bring in fundamental change of work and business model. Because of those applications and developments of Cloud Computing in the Radio and TV industry, the development trend of Cloud Computing must be one part of triple play.

By that time, TV users will enjoy the unlimited service which ere brought by Internet. All of those integrated information services will be spread to thousands of households just like water, electricity, coal gas and other infrastructures.

\section{The Cloud Storage Technology Based on Cloud Computing}

\section{Cloud Storage}

Cloud Storage is a new concept on the basis of the concept of Cloud Computing. Once this concept - Cloud Computing is proposed, it has been widely supported and concerned. From the view of technology, Cloud Computing is a large scale, "centralized” type of storage system. And at the same time, its storage system consists of different types of storage devices. And it is managed by special storage agents.

\section{The Analysis on Features of Cloud Storage}

Video Business is basic on the technology of Cloud Computing. It achieves magnanimity of content storage in low storage, funds a unified and sharing Resource Pool, and implements the unified support from video platform through Cloud Storage.

The features of Cloud Storage are as follows:

(1) High performance: it supports quick retrieval and block storage to magnanimity of files, current reading and writing to improve the I/O capability of system. It supports the distributed file system and distributed database.

(2) High reliability: it has multilevel fault-tolerant system, supporting data recovery between the nodes, high reliability of disk-level data and metadata. The system can recover itself, which is quite an obvious advantage.

(3) Intelligent management: the management involves the separation of point's domain, event alarms, performance statistics, remote maintenance, topology management, deployment automation.

(4) System compatibility: the function of pre-existing system has open interface, smooth and compatible access.

(5) High scalable: the system can manage dozens of PB data sizes, to expand capacity in classification in disk level, node level, domain level. 

Video surveillance,
IPTV and other contralized storage of systems
Application Interface Layer

Bace Management Layer

Storage Layer

Net(WAN or Internet), user authentication, rights management

Public API interface, application software, web service and etc.

\begin{tabular}{|c|c|c|}
\hline $\begin{array}{lr}\text { Cluster } & \text { system } \\
\text { distributed } & \text { file } \\
\text { system } & \text { net } \\
\text { computing } & \end{array}$ & $\begin{array}{l}\text { Content } \\
\text { distribution P2P } \\
\text { deduplication } \\
\text { data } \\
\text { compression }\end{array}$ & $\begin{array}{l}\text { Data encryption } \\
\text { Data backup } \\
\text { Data disaster recovery }\end{array}$ \\
\hline $\begin{array}{l}\text { Storage virtua } \\
\text { mnnitnrina main }\end{array}$ & centralized & management, \\
\hline
\end{tabular}

Storage device (NAS, FC, iSCSI)

Figure1: The system architecture model of cloud computing

\section{The System Structure of Cloud Storage}

Compared with traditional storage equipments, Cloud Storage is not only hardware but a complex system made up of network devices, storage devices, server, application software, public access interface, access network, client program etc. [5]Storage is the kernel of all parts, and it provides data storage service and business access service by application programs, this is a technical problem that has been studied a lot..

Cloud Storage is service-oriented. It offers the storage architecture to meet client's demand through hierarchical service-oriented architecture. Because of the different functions, it can be mostly divided into storage service layer, file service layer, application service layer. The structural model of Cloud Storage system is as shown in the figure 1.

\section{Summary}

With the development of cloud computing and cyber video technology, the construction of next generation broadcasting has provided a valuable opportunity for cloud network video' growth. Products based on the cloud cyber video service will come out, with more attractive characters. The practical application of cloud cyber video gives consumers more choices. Although there are some safety problems unsolved, cloud computing will be developed in the information safety area, more and more extensive in broadcast application. Cloud computing has now entered a new stage of development, and we can say that cloud computing is a new revolution of information era. Many experts think that cloud computing will change the Internet technology base, and will even affect the pattern of the whole industry. With the vigorous development of the media industry, all kinds of 
new media technology emerge in endlessly, and the development of high-tech help traditional business have the opportunity to be able to informatization, network.

\section{Acknowledgements}

Supported by the excellent young backbone teachers of Beijing city occupation college selection project.

\section{References}

[1] P. Salama, N.B. Shroff, E. J. Delp. Error Concealment in Encoded Video Streams [J].Signal Recovery Techniques for Image and Video Compression and Transmission, 1998:199-233.

[2] K. Shen, E. J.Delp. A Fast Algorithm for Video Parsing Using MPEG Compressed Sequences [J]. Proceedings of the IEEE International Conference on Image Processing, Oetober23-26, 1995, Washington, DC: 252-5.

[3] T. Silora. The MPEG-4 video standard verification model [J].IEEE Trans. Circuits Systems Video Technology, 1997, l: 19-31.

[4] F. Cuppens, A. Miege. Alert Correlation in a Cooperative Intrusion Detection Framework [J]. In: Proceedings of the 2002 IEEE Computer Society Symposium on Research in Security and Privacy, Berkeley, California, USA,2002: 202-215

[5] F. Cuppens, Fabien Autrel, Alexandre Mie ge. Correlation in an intrusion detection process. Proceedings SE'curite' des communications sur internet (SECI02); Paris, France: IEEE Computer society [J]. 2002: 204-215 